\title{
Visualization of coherent structures in a supersonic flat-plate boundary layer
}

\author{
HE Lin*, YI ShiHe, ZHAO YuXin, TIAN LiFeng \& CHEN Zhi \\ College of Aerospace and Material Engineering, National University of Defense Technology, Changsha 410073, China
}

Received August 2, 2010; accepted October 19, 2010

Flow in a flat-plate zero-pressure-gradient boundary layer at Mach 3 was visualized via nanoparticle-based planar laser scattering (NPLS). Coherent structures such as an individual hairpin vortex and hairpin packet were identified in the streamwise-wallnormal plane on the basis of the now accepted hairpin model. $\Lambda$-shaped vortices were found in a staggered pattern in the streamwise-spanwise plane, which indicated H-type transition in the present experiments. This is the direct evidence (in the form of flow visualization) of such coherent structures in a supersonic boundary layer. A series of NPLS images taken in streamwise-spanwise planes at different heights is presented, and the three-dimensional structures of the supersonic boundary layer agree well with the hairpin model.

supersonic boundary layer, flow visualization, NPLS, coherent structures, $\Lambda$-vortices

Citation: He L, Yi S H, Zhao Y X, et al. Visualization of coherent structures in a supersonic flat-plate boundary layer. Chinese Sci Bull, 2011, 56: 489-494, doi: $10.1007 / \mathrm{s} 11434-010-4312-\mathrm{z}$

Over the past few decades, a large amount of work has been devoted to the study of coherent structures in wall-bounded flows, especially in the turbulent boundary layer. Although coherent structures continue to be one of the outstanding unsolved questions in the study of turbulence, there is general agreement that hairpin vortices, streamwise vortices and streaks, as well as sweeps and ejections, constitute the main coherent structures in the boundary layer. Previous laboratory and numerical experiments provide convincing evidence for the presence of the hairpin vortex, which was first proposed by Theodorsen [1] and later confirmed experimentally through flow visualization by Head and Bandyopadhyay [2]. The idealized hairpin model is characterized by its three-dimensional structure, which consists of a pair of counter-rotating quasi-streamwise vortices (two legs) and a head segment joined by two necks to the legs (Figure 1(a)). However, Choi and Guezennec reported the presence of asymmetric hairpins or cane-like vortices rather than symmetric ones in their studies [3,4]. In addition to an indiv idual hairpin vortex, a new model termed "hairpin packets"

\footnotetext{
*Corresponding author (email: helin.101sys@gmail.com)
}

was proposed by Adrian et al. in their particle image velocimetry (PIV) study of a turbulent boundary layer [5], where multiple hairpin vortices were observed to travel in groups. An idealized packet of hairpins is shown in Figure 1(b). Evidence of hairpin packets in the outer region of turbulent channel flow was also observed through PIV measurements by Christensen and Adrian [6].

In comparison with the case for incompressible flows, there have been few studies on coherent structures in supersonic and hypersonic flows. Ringuette et al. [7] utilized a direct numerical simulation (DNS) database of Mach 3 turbulent boundary layers and found large-scale coherent structures similar to those observed in experiments under both supersonic and incompressible conditions. Quasistreamwise vortices or vortex pairs were detected in the wall region of a turbulent boundary layer at Mach 4.5 by Huang et al. [8], who also used a DNS database. In experimental studies, coherent structures in a supersonic turbulent boundary layer were visualized by Smiths et al. [9] employing different methods. Using the recently developed nanoparticle-based planar laser scattering (NPLS) technique, the interaction between an oblique shock wave and turbu- 

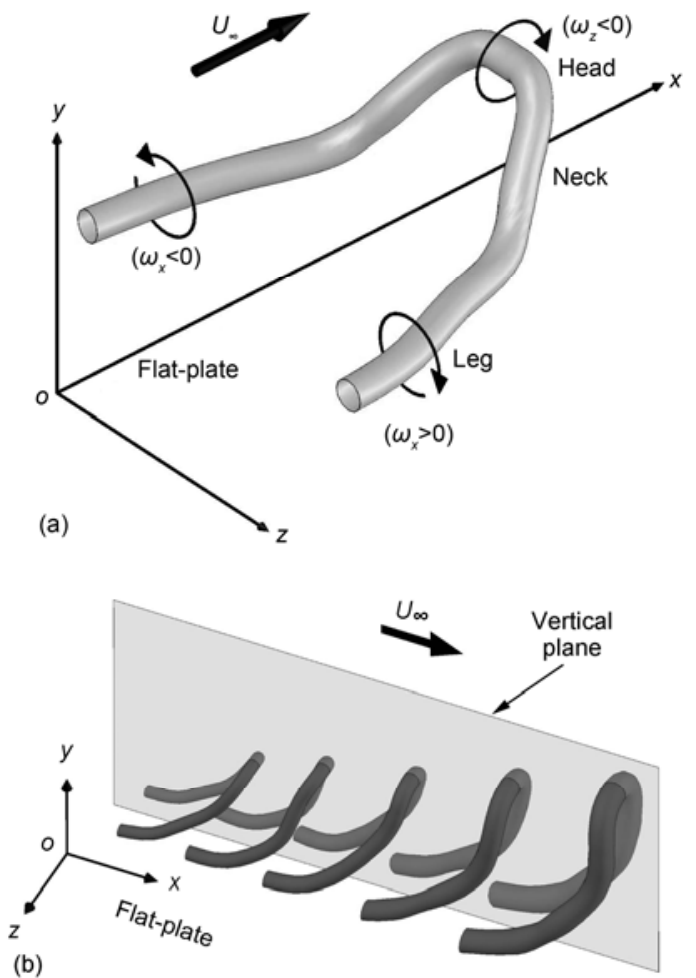

Figure 1 Schematic of the idealized hairpin model. (a) Individual hairpin; (b) hairpin packet.

lent boundary layer was visualized by Zhao et al. [10], as well as the influence of a large vortex of the mixing layer on an oblique shock wave. The density field measurement of a supersonic mixing layer was also studied using this method. The approximate reconstruction of the three-dimensional density field and multiresolution analysis of the density fluctuation have been carried out $[11,12]$. The structures of supersonic turbulent boundary layers were reviewed by Smits and Dussauge [13].

In most previous experimental studies, hairpin vortices were detected using PIV or hot-wire database; however, direct visual evidence of the existence of hairpin vortices in supersonic flows is still lacking. Taking advantage of the good performance of the NPLS technique in supersonic flow visualization, the aim of the current study is to apply this technique to visualizing coherent structures in a flatplate zero-pressure-gradient boundary layer at Mach 3 . Based on the hairpin model described in previous works, we attempted to identify individual hairpin vortices as well as hairpin packets in NPLS flow images.

\section{Apparatus and experimental techniques}

\subsection{Flow facility}

Experiments were performed in KD-03 indraft supersonic wind tunnel (Figure 2). The test section dimensions are 120 $\mathrm{mm} \times 100 \mathrm{~mm}$, and optical glasses are embedded in each side of the test section to provide optical access from four sides. The flow parameters in the present experiments are given in Table 1.

The boundary layer was generated by a horizontal flat plate placed $30 \mathrm{~mm}$ above the floor of the test section. Images of the boundary layer were obtained in streamwisewall-normal (side view) and streamwise-spanwise (top view) planes. For the side view, the laser sheet was perpendicular to the wall along the centerline of the plate, and for the top view, the laser sheet was parallel to the wall at different heights. A charge-coupled device (CCD) camera was set perpendicular to the laser sheet in each view. Schematic representations of the experimental arrangements are shown in Figure 3(a) and (b), respectively.

\subsection{NPLS technique}

The flow structures of the boundary layer were visualized using the NPLS technique. Nanoparticles are used asracer particles and a pulse planar laser is used for illumination in NPLS; by recording images of particles in the flowfield with the CCD camera, imaging of supersonic flow with high spatiotemporal resolution is realized. Theflow-following ability of nanoparticles in supersonic flow has been validated in experiments, and high spatiotemporal resolution images of the shock wave, expansion, Mach disk, sliding line and mixing layer have been obtained with NPLS [14-17]. A detailed description of this technique has been given by Zhao et al. [18]. A Q-switched Nd:YAG laser is used as the light source. The pulse energy output to the test section is $350 \mathrm{~mJ}$ for a pulse width of $6 \mathrm{~ns}$ and wavelength of $532 \mathrm{~nm}$. The laser beam is oriented by an articulated arm and focused as a uniform sheet by a cylindrical lens. The recording system is an interline transfer CCD camera. The CCD array has $2000 \times 2000$ pixels with 4096 grayscale grades.

Table 1 Experimental parameters

\begin{tabular}{ccccc}
\hline$M_{\infty}$ & $P_{0}(\mathrm{~Pa})$ & $T_{0}(\mathrm{~K})$ & $\operatorname{Re}(1 / \mathrm{m})$ & $U_{\infty}(\mathrm{m} / \mathrm{s})$ \\
\hline 3.0 & $1.01 \times 10^{5}$ & 300 & $7.7 \times 10^{6}$ & 620 \\
\hline
\end{tabular}

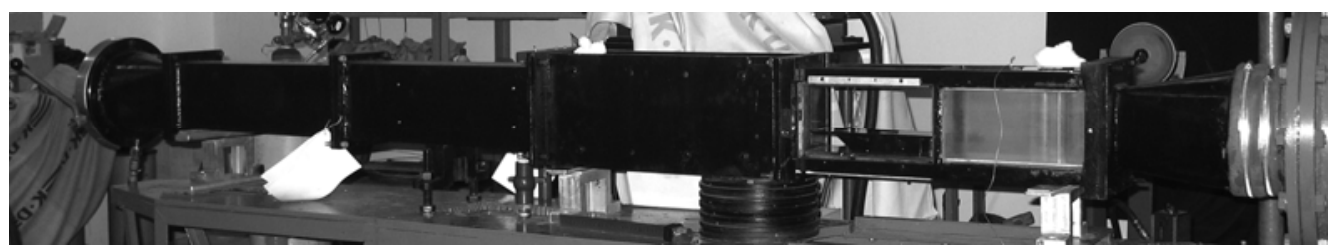

Figure 2 KD-03 supersonic wind tunnel. 

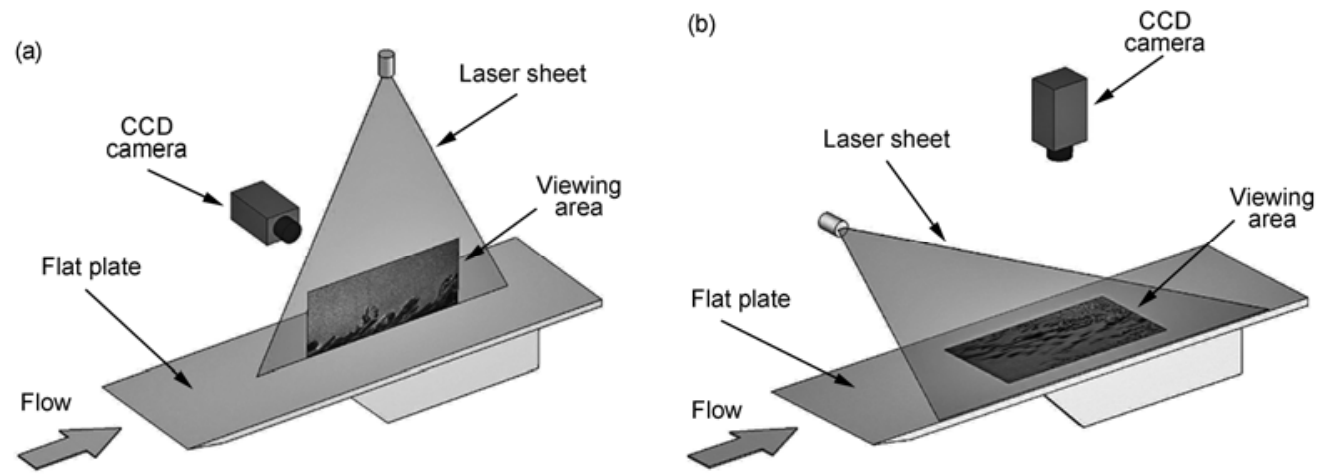

Figure 3 Experimental arrangement. (a) Side view; (b) top view.

\section{Coherent structures of the boundary layer in the streamwise-wall-normal plane}

A typical NPLS image of the flat-plate boundary layer in the streamwise-wall-normal plane is shown in Figure 4. The grayscale of the NPLS image is proportional to the local density as reported by Tian et al. [17]. Thus, the dark (low grayscale) regions in Figure 4 should represent the boundary layer, where the local density is low. As shown in Figure 4 , the spatial structure of a flat-plate boundary layer in natural transition can be identified clearly. In the upstream region, the boundary layer is laminar with linear growth; further downstream, wavelike structures can be observed, and the flow is unstable. In the downstream region, the breaking down of large-scale structures reveals transition from laminar to turbulent flow.

Successive NPLS images of the boundary layer are shown in Figure 5. Compared with the two images, spanwise vortices with clockwise rotation, indicated by white arrows, are identified in the streamwise-wall-normal plane. An inclined structure is observed upstream of each spanwise vortex connecting the vortex core to the wall. On the basis of the idealized model of an individual hairpin (Figure 1(a)), the hairpin vortex in the streamwise-wallnormal plane can be identified by (i) a spanwise vortex core of the head rotating clockwise and (ii) an inclination to the flow direction upstream of the spanwise vortex and more tangent to the wall as the wall is approached. These features are consistent with the structures mentioned above, and two individual hairpins labeled 1 and 2 are identified clearly in
Figure 5. Hairpin 1 inclines at a mean angle of approximately $24^{\circ}$ relative to the wall, and has a length of $5.1 \mathrm{~mm}$ in the streamwise direction and a height of $2.4 \mathrm{~mm}$. Owing to being more downstream, hairpin 2 is slightly larger, and has a length of $5.8 \mathrm{~mm}$ and height of $3.2 \mathrm{~mm}$. The angle of inclination of hairpin 2 is small close to the wall and increases to $47^{\circ}$ toward the head of the hairpin. After a $5 \mu \mathrm{s}$ delay (Figure 5(b)), these hairpins grow as they move downstream with their heads lifting away from the wall.

A hairpin packet can be identified as consisting of multiple hairpins as shown in Figure 6. Individual hairpins (indicated by white arrows) within this group propagate at nearly the same streamwise velocity owing to a relatively constant streamwise position with respect to each other after $5 \mu \mathrm{s}$ delay. However, hairpin 2 is found to move more quickly than hairpin 3. The characteristic growth angle defined as the angle of the envelope of the packet is less than $9^{\circ}$, which is smaller than the inclination of individual hairpins ranging from $15^{\circ}$ to $50^{\circ}$. A similar conclusion can be drawn from the foregoing results as Adrian et al. concluded for incompressible flows [5]. Owing to the full complexity of real flows, no hairpin packet is found in a regular array as shown in Figure 1(b).

\section{Coherent structures in the streamwise-span- wise plane}

The coherent structures of the boundary in the streamwisespanwise plane are shown in Figure 7. In this case, the laser

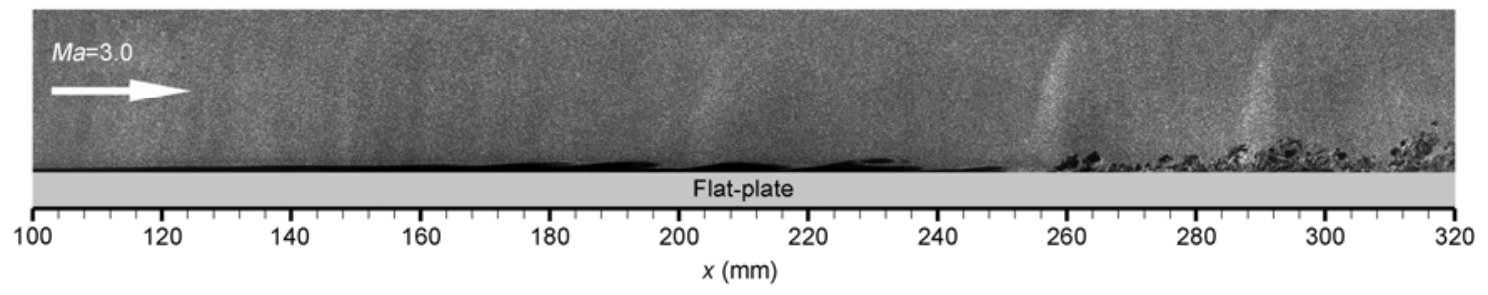

Figure 4 NPLS image of the flat-plate boundary layer in the streamwise-wall-normal plane. The coordinate system is chosen with the origin at the leading edge centerline of the plate, and the coordinates $x, y$ and $z$ correspond to the streamwise, wall-normal and spanwise directions, respectively. 


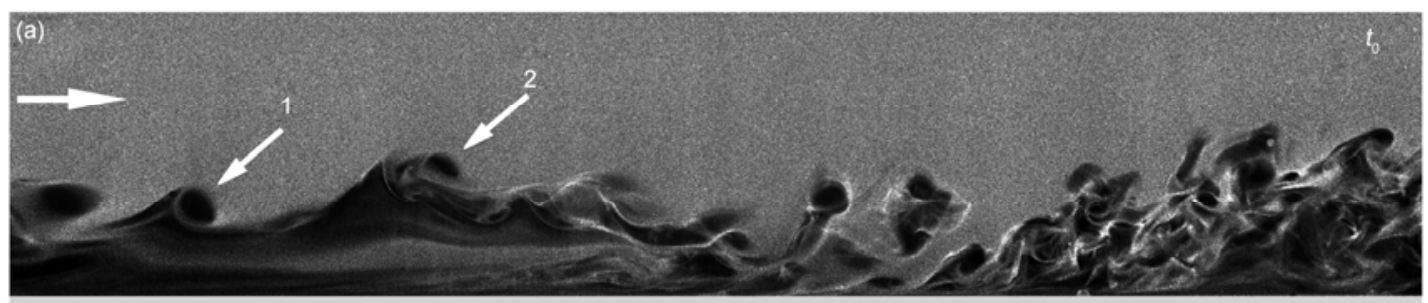

Flat-plate

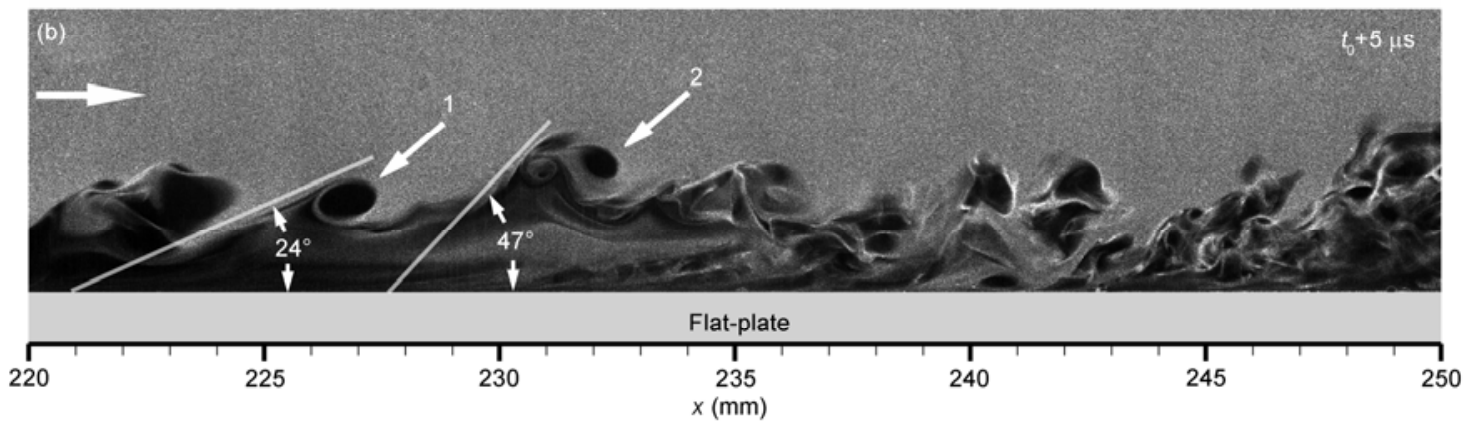

Figure 5 Successive NPLS images of individual hairpin vortices in the streamwise-wall-normal plane.

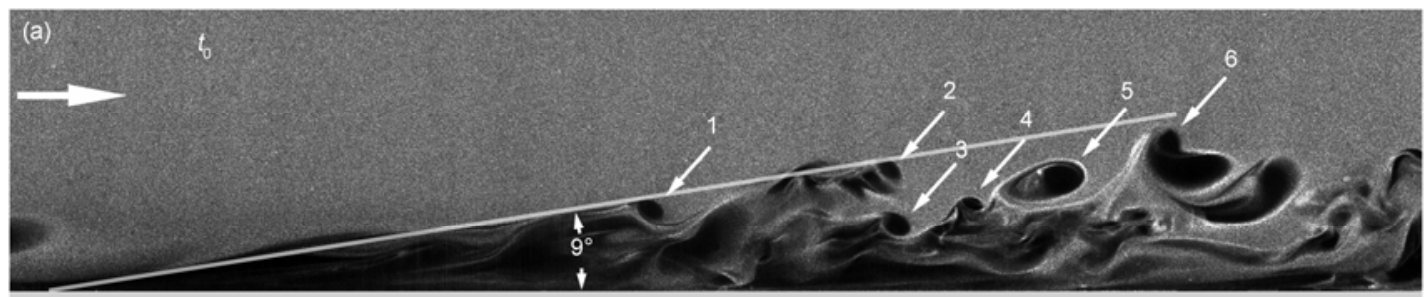

Flat-plate
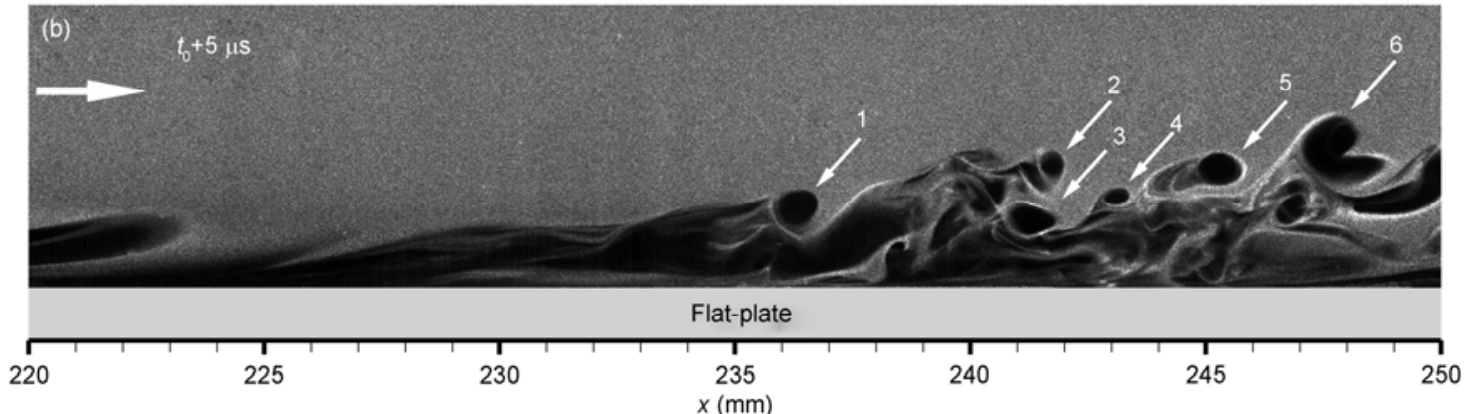

Figure 6 Successive NPLS images of hairpin packets in the streamwise-wall-normal plane.

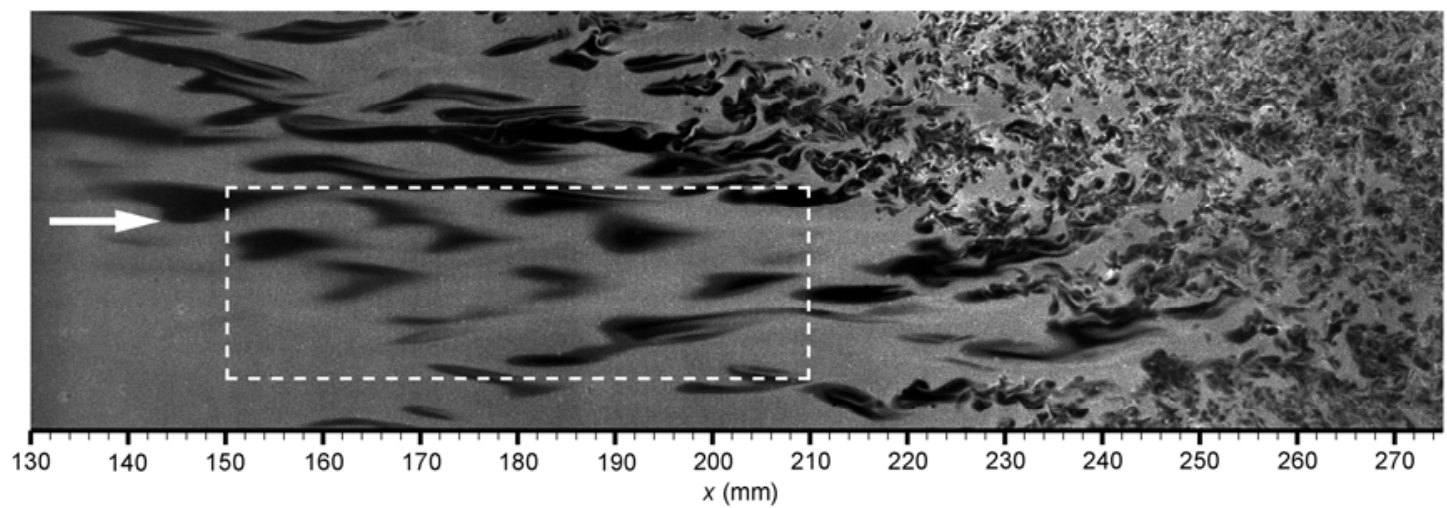

Figure 7 NPLS image of the boundary layer in the streamwise-spanwise plane at a height of $1.5 \mathrm{~mm}$ above the wall. 
sheet was parallel to the wall with a height of approximately $1.5 \mathrm{~mm}$. Along the streamwise direction, three-dimensional structures due to the interactions of flow instability can be identified. Further downstream, there is laminar-turbulent transition, and large-scale three-dimensional structures break down into smaller eddies.

As shown in Figure 7, hairpin vortices are elongated in the form of a $\Lambda$-shape owing to the increase in the Reynolds number. However, similar structures are better described as horseshoe vortices or $\Omega$-shaped vortices at low Reynolds numbers $\left(R e_{\theta}<800\right)$ as reported by Head and Bandyopadhyay [2]. In the present case, the mean length of these $\Lambda$-vortices is about $9.7 \mathrm{~mm}$ in the streamwise direction, and the mean width is about $3.9 \mathrm{~mm}$ in the spanwise direction.
Nevertheless, owing to the three-dimensional structure of hairpin vortices, the size differs in the streamwise-spanwise plane at different heights. As indicated by the white dashed rectangle, a staggered order of $\Lambda$-vortices in the streamwise direction is identified clearly, as observed in experiments under incompressible condition by Knap and Roache [19]. According to the physical mechanisms of the boundarylayer transition summarized by Kachanov [20], these structures provide direct visual evidence of the existence of H-type transition (in accordance with Herbert's theory [21]) in the present supersonic boundary layer.

Coherent structures of the boundary layer in the streamwise-spanwise plane at different heights are shown in Figure 8. As shown in Figure 8(a), boundary-layer structures

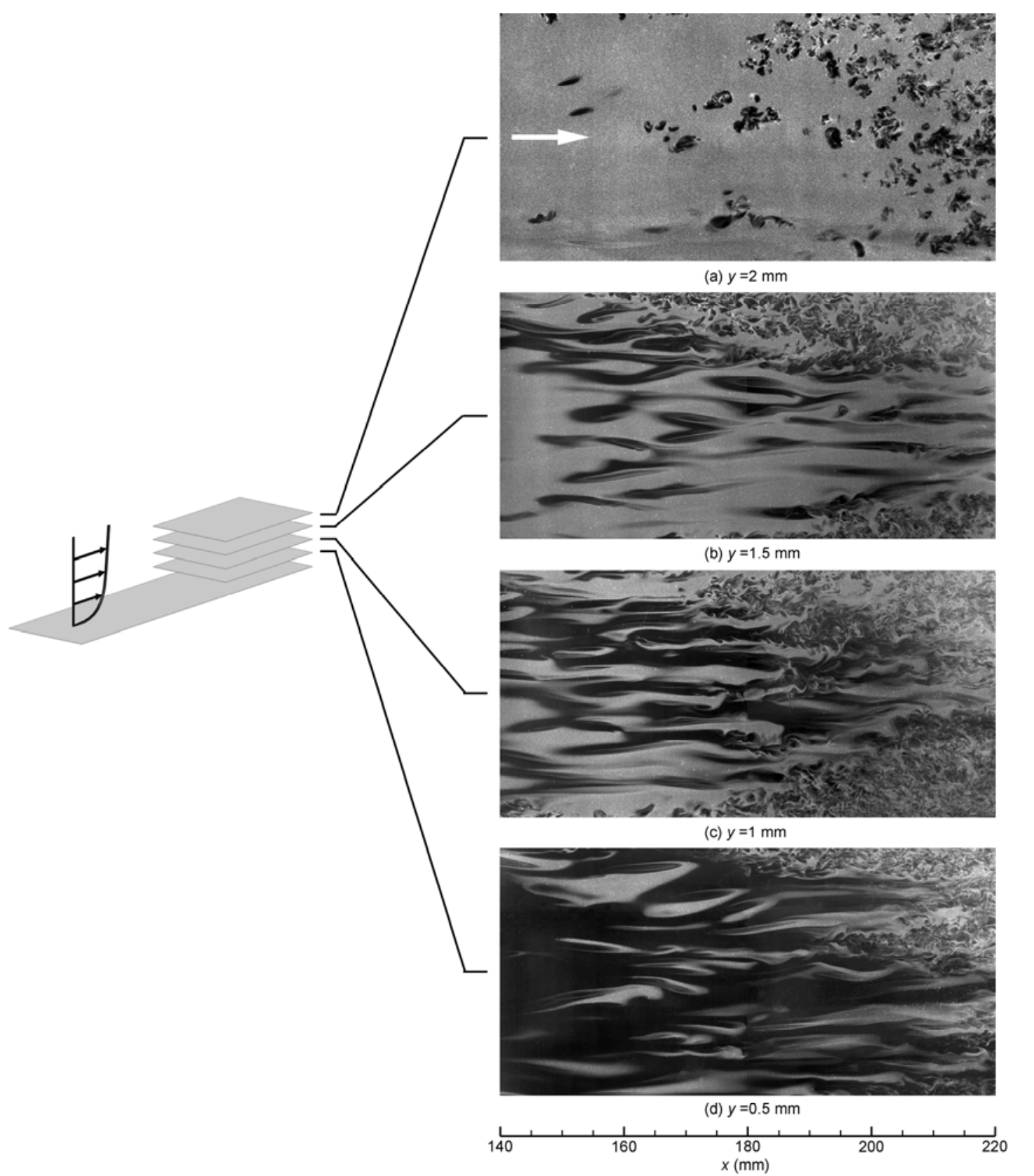

Figure 8 NPLS images of the boundary layer in the streamwise-spanwise plane at different heights. These four images were not taken simultaneously. 
can only be identified where the local thickness of the boundary layer is greater than $2 \mathrm{~mm}$. At a lower height, $\Lambda$-vortices are found in a staggered pattern as in Figure 8(b). In addition to symmetric hairpin vortices, asymmetric hairpin and cane-like (or one-legged) hairpin vortices are also found; similar structures in incompressible boundary layer were reported by Choi and Guezennec [3,4]. Based on the idealized hairpin model (Figure 1), two legs are connected to the head of a hairpin, and thus, a $\Lambda$-shaped vortex can be identified only at the height of the head. Comparing the four NPLS images in Figure 8, we infer that the mean height of hairpin heads is approximately $1.5 \mathrm{~mm}$ in the present experiments. Below the heads of the hairpins, low-speed (also low-density for the compressible boundary layer) streaks are generated by the induction of the vortex legs, which pump low-speed fluid upward. Figure 8(c) shows dark streaks along the direction of the hairpin legs. Close to the wall, high-speed (high-density) streaks are generated also by the induction of the vortex legs, and thus, light streaks are identified as in Figure 8(d). However, the light streaks are found to be oriented upstream under the hairpin heads, where the back-induction of the head is strong.

\section{Conclusions}

Flow visualization of a flat-plate zero-pressure-gradient boundary layer at Mach 3 is performed, and the coherent structures in streamwise-wall-normal and streamwisespanwise planes are shown in the NPLS images. Individual hairpins as well as a hairpin packet are identified in the streamwise-wall-normal plane. Owing to the high Reynolds numbers, $\Lambda$-shaped vortices rather than horseshoe-shaped vortices or $\Omega$-shaped vortices are observed in the streamwisespanwise plane. There is a staggered pattern of $\Lambda$-vortices, indicating $\mathrm{H}$-type transition, in the present experiments. Direct visual evidence of such coherent structures in the supersonic boundary is shown in the form of flow visualization. Three-dimensional structures are recognized in a series of NPLS images of the streamwise-spanwise planes at different heights and agree well with the idealized hairpin model.

This work was supported by the National Basic Research Program of China (2009CB724100).
1 Theodorsen T. Mechanism of turbulence. In: Proceedings of the Second Midwestern Conference on Fluid Mechanics, Ohio State University, Columbus, March 17-19, 1952

2 Head M R, Bandyopadhyay P. New aspects of turbulent boundarylayer structure. J Fluid Mech, 1981, 107: 297-338

3 Choi W C, Guezennec Y G. On the asymmetry of structures in turbulent boundary layers. Phys Fluids, 1989, 2: 628-630

4 Guezennec Y, Piomelli U, Kim J. On the shape and dynamics of wall structure in turbulent channel flow. Phys Fluids A, 1989, 1: 764-766

5 Adrian R J, Meinhart C D, Tomkins C D. Vortex organization in the outer region of the turbulent boundary layer. J Fluid Mech, 2000, 422: $1-54$

6 Christensen K T, Adrian R J. Statistical evidence of hairpin vortex packets in wall turbulence. J Fluid Mech, 2001, 431: 433-443

7 Ringuette M R, Wu M W, Martin M P. Coherent structures in direct numerical simulation of turbulent boundary layers at Mach 3. J Fluid Mech, 2008, 594: 59-69

8 Huang Z F, Zhou H, Luo J S. The investigation of coherent structures in the wall region of a supersonic turbulent boundary layer based on DNS database. Sci China Ser G-Phys Mech Astron, 2007, 50: 348356

9 Smith M W, Smits A J. Visualization of the structure of supersonic turbulent boundary layers. Exp Fluids, 1995, 18: 388-302

10 Zhao Y X, Yi S H, He L, et al. The experiment study of interaction between shock wave and turbulence. Chinese Sci Bull, 2007, 52: 1297-1031

11 Zhao Y X, Yi S H, Tian L F, et al. Density field measurement and approximate reconstruction of supersonic mixing layer. Chinese Sci Bull, 2010, 55: 2004-2009

12 Zhao Y X, Yi S H, Tian L F, et al. Multiresolution analysis of density fluctuation in supersonic mixing layer. Sci China-Tech Sci, 2010, 53: 584-591

13 Smits A J, Dussauge J P. Turbulent Shear Layers in Supersonic Flow. 2nd ed. New York: Springer, 2006

14 Zhao Y X, Yi S H, Tian L F, et al. Nano-tracer planar laser scattering. In: Proceedings of the 13th National Symposium on Shock Wave and Shock Tube Technology, 2008. 323-330

15 Tian L F, Yi S H, Zhao Y X, et al. Flow visualization of supersonic around a concave optical bow cap model of warhead. J Exp Fluid Mech, 2009, 23: 15-17

16 Zhao Y X, Yi S H, He L, et al. The fractal measurement of experimental images of supersonic turbulent mixing layer. Sci China Ser G-Phys Mech Astron, 2008, 51: 1134-1143

17 Tian L F, Yi S H, Zhao Y X, et al. Study of density field measurement based on NPLS technique in supersonic flow. Sci China Ser G-Phys Mech Astron, 2009, 52: 1357-1363

18 Zhao Y X, Yi S H, Tian L F, et al. Supersonic flow imaging via nanoparticles. Sci China Ser E-Tech Sci, 2009, 52: 3640-3648

19 Knapp C F, Roache P J. A combined visual and hot-wire anemometer investigation of boundary-layer transition. AIAA J, 1968, 6: 29-36

20 Kachanov Y S. Physical mechanisms of laminar-boundary-layer transition. Ann Rev Fluid Mech, 1994, 26: 411-482

21 Herbert T. Secondary instability of plane channel flow to subharmonic three-dimensional disturbances. Phys Fluids 1983, 26: 871874

Open Access This article is distributed under the terms of the Creative Commons Attribution License which permits any use, distribution, and reproduction in any medium, provided the original author(s) and source are credited. 